\title{
TRAFFIC ENTRY JUDGMENTS BY AGING DRIVERS
}

\author{
Nicole Skaar \\ Matthew Rizzo \\ Laura Stierman \\ Colleges of Medicine, Engineering, and Public Health \\ Public Health Center \\ University of Iowa \\ Iowa City, Iowa, USA \\ E-mail: nicole-skaar@uiowa.edu
}

\begin{abstract}
Summary: We hypothesized that older, neurologically normal drivers would compensate appropriately for their slower abilities by choosing larger gaps when entering traffic. To test this we used an instrumented vehicle and radar gun to study 18 legally licensed, neurologically normal drivers ranging from 22 to 72 years old. Drivers were asked to press a button to mark the last possible moment they would cross the road in front of an oncoming vehicle. We measured speed and distance of the oncoming vehicles and calculated time-to-contact (TTC). The older drivers made more conservative gap acceptance decisions based on higher TTC than younger drivers. This pilot study identified trends in effects of age upon traffic entry judgments, suggesting that neurologically normal older drivers are more conservative when deciding to enter traffic than younger drivers.
\end{abstract}

\section{INTRODUCTION}

Automobile driving is a complex task requiring the coordination of multiple cognitive processes, including visuomotor, attentional and decision-making skills. Impairments of these abilities and general slowing may place aging drivers at greater risk for crashes, particularly during maneuvers across traffic streams (e.g., Lyles and Staplin, 1991). Hills and Johnson (1980) found that older drivers maintain a constant distance gap while younger drivers compensate for speed by keeping a constant time gap. Staplin (1995) reported that older drivers relied on distance cues while making gap acceptance judgements during left turns in front of oncoming traffic. The older drivers chose the same "minimum safe gap" distance for vehicles traveling at $48 \mathrm{~km} / \mathrm{h}$ and $96 \mathrm{~km} / \mathrm{h}$, increasing the possibility of a crash with vehicles traveling at higher speeds. Drivers who maintain a constant gap acceptance distance regardless of speed may be more likely to pull out than drivers who adjust for speed in unsafe circumstances, resulting in crashes with vehicles traveling at high velocities.

Yet, other studies suggest older drivers take fewer risks than younger drivers, as Boyce and Geller (2000) found in an instrumented vehicle field test. While their study did not specifically address gap acceptance for entering traffic, results showed that younger individuals drove faster and followed a lead vehicle more closely. In line with this more conservative behavior of older drivers, we hypothesized that neurologically normal older drivers would compensate appropriately for their slower processing abilities by choosing larger gaps when entering traffic. Also, they would not show a constant gap acceptance distance for varying speeds of oncoming traffic. 


\section{METHODS}

\section{Participants}

We studied 18 legally licensed, neurologically normal drivers (range 22-78 years, mean=49.44 [4.40]). Eight drivers were older (range 60-78 years, mean=65.75 [2.13]) and 10 younger (range 22-57 years, mean=36.4 [4.51]). All drivers participated in experiments conducted in a multipurpose field research vehicle, known as ARGOS, in strict accord with federal human subjects' protection guidelines.

\section{Instrumented Vehicle}

The Automobile for Research in Ergonomics and Safety (ARGOS) is a mobile laboratory comprised of a mid-sized vehicle with extensive hidden instrumentation. ARGOS was designed to examine quantitative indices of driving performance in different populations, including potentially unfit drivers with medical impairments.

\section{Procedures}

Drivers entered the vehicle in the hospital parking area. After being familiarized with the controls of ARGOS, they drove approximately 3 miles of city driving to become proficient in the "handling" of ARGOS. The experimenter sat in the passenger seat throughout the drive and in subsequent testing of gap acceptance behavior.

Gap acceptance behavior was tested with the driver parked in an empty driveway perpendicular to a busy 4-lane highway. This safety precaution allowed us to assess driver performance without exposing the driver to a potential vehicular collision.

We measured speed, distance and time-to-contact (TTC) of each oncoming vehicle with the Stalker LIDAR system (Plano, TX). Stalker LIDAR is a semi-conductor laser device that measures the speed, distance and direction vehicles are travelling relative to the device. The LIDAR was pointed directly at an oncoming vehicle by the experimenter sitting in the passenger seat (Figure 1). The laser beam was directed at the license plate of the oncoming vehicle to ensure accurate results. Oncoming traffic rounding a curve entered the view of the experimenter and participant approximately $1000 \mathrm{ft}$ down the road.

Each driver was asked to press a button to mark the last possible moment he or she would cross the road in front of a specified oncoming vehicle. Each driver performed this judgment until 10 trials were completed. Data streamed on-line from the LIDAR device to a lap top computer for quality assessment, artifact detection, and calculation of TTC for each button press. Trials were rejected when a passing vehicle unexpectedly occluded the LIDAR beam or when a participant had mechanical difficulty depressing the button.

We also obtained independent estimates of actual time it took each driver to cross the road where the experiment was conducted. When the road was clear of traffic, each driver crossed the roadway three times. We calculated average safety cushion (seconds) as the difference between the average TTC and average time to cross the roadway in each driver. 


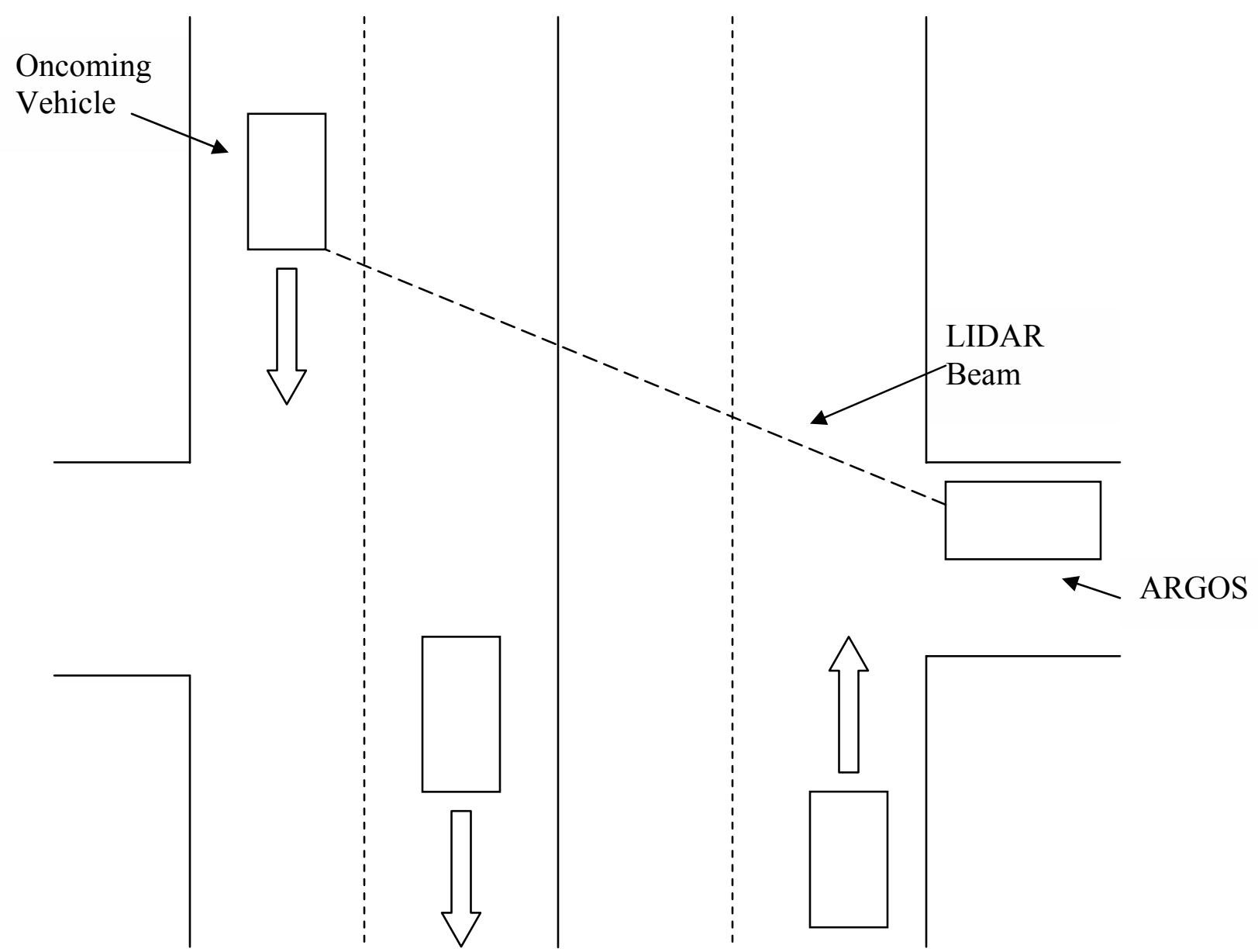

Figure 1. Diagram showing the layout of the area in which the experiment took place

\section{RESULTS}

The data in this pilot study showed trends in the direction of the hypothesis that older drivers take a more conservative approach to entering traffic. Table 1 summarizes the mean, median and standard deviation for TTC, time to cross the roadway, and cushion. Older drivers made more conservative gap acceptance decisions based on a median TTC (for last possible moment to cross in front of oncoming traffic) that was approximately 0.8 seconds greater than that of the younger drivers $(\mathrm{P}=0.21)$. Older drivers took only 0.2 seconds longer than younger drivers to cross the roadway $(\mathrm{P}=0.82)$, but showed a 1.3 second greater cushion than younger drivers $(\mathrm{P}=0.17)$. The ratio of mean cushion to average time to cross the road was $50 \%$ larger in the older drivers. Only one driver, a younger driver, had a slightly negative cushion (-0.47s), compatible with aggressive maneuvering: to avert contact, an oncoming car would need to brake or steer. Statistical comparisons used the Wilcoxon Rank Sum test. 
Table 1. Mean, Median and Standard Deviation (SD) For Each Age for Each Dependent Measure

\begin{tabular}{|cccccccccc|c|}
\hline & \multicolumn{3}{c}{} & \multicolumn{3}{c}{ Time to Contact(sec) } & \multicolumn{2}{c}{ Time to Cross(sec) } & & \multicolumn{2}{c|}{ Cushion(sec) } \\
Age Group & Mean & Median & SD & Mean & Median & SD & Mean & Median & SD \\
\hline Younger & 6.52 & 6.67 & 1.45 & 4.8 & 4.75 & 0.39 & 1.71 & 1.79 & 1.39 \\
Older & 7.44 & 7.45 & 1.21 & 4.82 & 4.95 & 0.64 & 2.62 & 3.08 & 1.54 \\
\hline
\end{tabular}

We used Spearman correlations to analyze speed and gap distance independent of TTC for each group across the 10 trials. This analysis showed that the average correlation between gap distance and the speed of oncoming cars was not significantly different across age groups (average $\mathrm{r}=0.16$ for older, average $\mathrm{r}=0.35$ for younger, $\mathrm{P}=0.216$ ) suggesting a similar strategy in both groups for adjusting gap distance. Figure 2 summarizes this similarity between older and younger drivers.

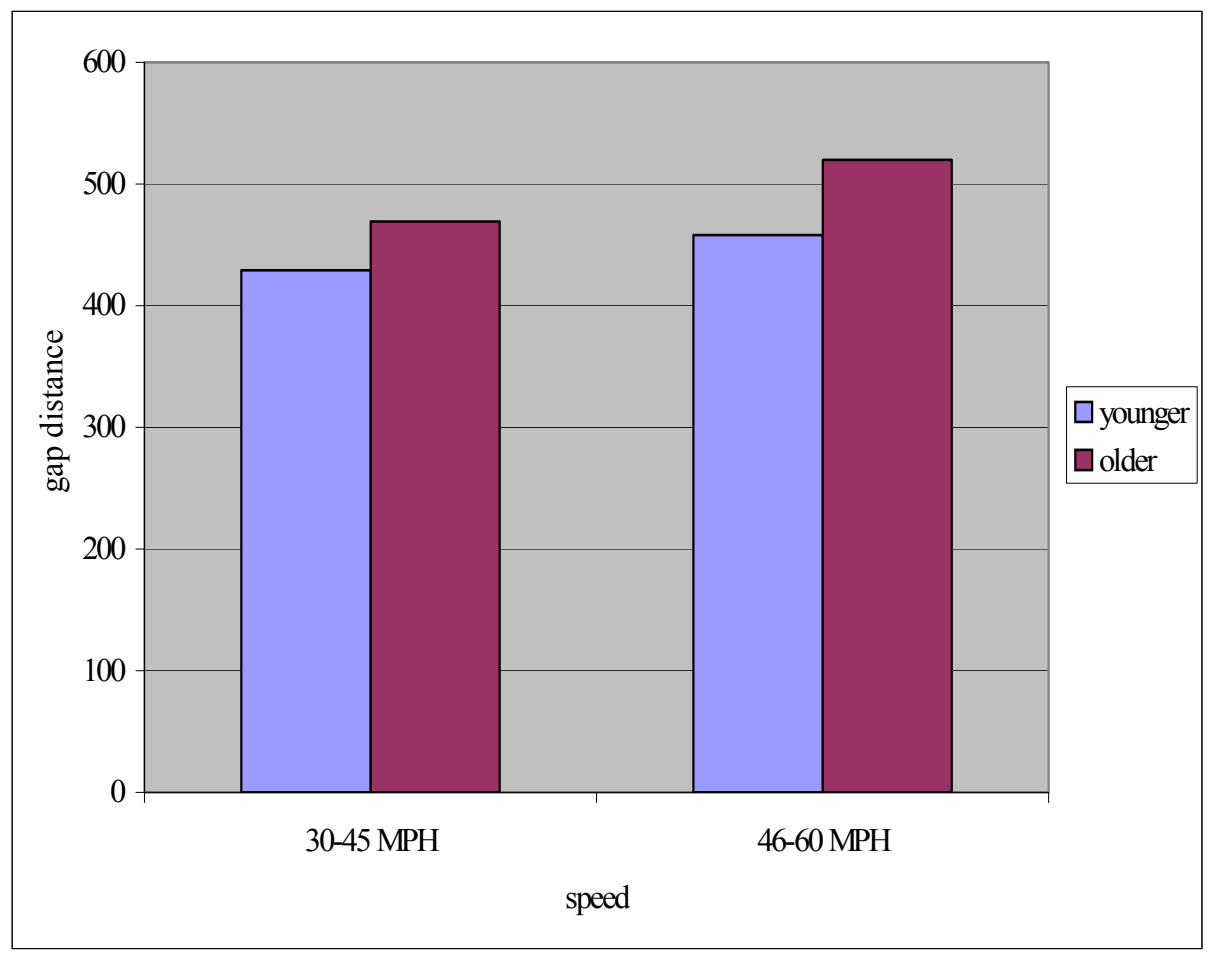

Figure 2. Gap Distance Judgements as a Function of Speed in Older and Younger Drivers

\section{CONCLUSIONS}

Safe drivers tend to adjust traffic-entry decisions for oncoming vehicle speed to minimize the risk of crashes. These adjustments are key to carrying out driving maneuvers such as merging, entering traffic from the side of a road or from a driveway, or while making turns without colliding with other vehicles. Left-hand turn collisions are more common among older drivers (NHTSA, 2001) and may be due to impaired gap acceptance criteria in decisions to turn ahead of or cross oncoming traffic. In two-vehicle crashes involving one older driver and one younger 
driver, $26 \%$ of the crashes involved the older driver turning left across traffic, 6 times as often as the younger driver (NHTSA, 2001).

Previous studies suggested that normal older drivers were at greater risk for a crash due to constant gap acceptance distances (Hills and Johnson, 1980; Staplin, 1995). These studies analyzed speed and distance without considering TTC and assumed that older drivers made unsafe judgments when they failed to adjust gap distance for speed. The current study addresses gap acceptance decisions based on TTC, speed and distance. The trends in this pilot study suggest that older drivers adjust gap distance for speed as younger drivers do, while allowing a larger cushion to cross the road safely.

The trends also suggest that neurologically normal older drivers are more conservative than younger drivers when deciding to enter traffic, compatible with Alexander et al. (2002), who reported that older drivers show more conservative gap judgments in right turns across streams of simulated traffic. The older drivers required more time to cross the road, but accepted larger gaps, reducing the probability of a traffic incident compared to younger drivers. Like the older drivers in this pilot study, they were apparently aware of their slowed ability to operate vehicle controls and cross the road, and compensated appropriately by choosing larger gaps and safety cushions.

The results of the current pilot study provide a baseline for interpreting decisions to enter traffic in drivers with cognitive decline and impaired self-awareness due to Alzheimer's disease, stroke, overmedication, and other conditions associated with advancing age. Future studies could address how disorders of vision, attention and cognition (Owsley, et al., 1991; Ball et al., 1993; Rizzo et al., 2001), and certain personality traits (Wilson, 1990; Furnham and Saipe, 1993; Arnett, 1996) affect decisions to enter traffic.

\section{REFERENCES}

Arnett, J.J. 1996. Sensation seeking, aggressiveness and adolescent reckless behavior. Personality and Individual Differences, 20: 693-702.

Alexander, J., Barham P., \& Black I. 2002. Factors influencing the probability of an incident at a junction: Results from an interactive driving simulator. Accident Analysis and Prevention, 34 (6): 779-792.

Ball, K., Owsley, C., Sloane, M. E., et al. 1993. Visual attention problems as a predictor of vehicle crashes in older drivers. Investigative Ophthalmology and Visual Science, 34: 31103123.

Ball, K. K., Beard, B. L., Roenker, D. L., et al. 1988. Age and visual search: Expanding the useful field of view. Journal of the Optical Society of America, A; 5:2210-2219.

Boyce, T., \& Geller, E. 2000. An instrumented vehicle assessment of problem behavior and driving style: Do younger males really take more risks? Accident Analysis and Prevention, 34 (1): 51-64.

Furnham, A., \& Saipe, J. 1993. Personality correlates of convicted drivers. Personality and individual differences, 14 (2): 329-336. 
Hills, B.L., \& Johnson, L. 1980. Speed and Minimum Gap Acceptance Judgements at Two Rural Junctions. Report SR515. Crowthorne, Berkshire, England: Department of the Environment, Transport and Road Research Laboratory.

Lyles, R. W., \& Staplin, L. 1991. Age differences in motion perception and specific traffic maneuver problems. Transportation Research Record 1325: 23-33.

National Highway and Traffic Safety Administration. 2001. Traffic Safety Facts. Report DOT HS 809475.

Owsley, C., Ball, K., Sloane, M. E., et al. 1991. Visual/cognitive correlates of vehicle accidents in older drivers. Psychology and Aging, 6:403-415.

Staplin, L. 1995. Simulator and field measures of driver age differences in left-turn gap judgments. Transportation Research Record 1485: 49-55.

Wilson, R. J. 1990. The relationship between seat belt non-use to personality, lifestyle and driving record. Health Education Research: Theory and Practice, 5 (2): 175-185.

Supported by NIA AG15071 and NIA AG17717 